\title{
Decoherence and kinetic processes in quantum nanosystems
}

\author{
Massimiliano Esposito, Pierre Gaspard* \\ Center for Nonlinear Phenomena and Complex Systems, Université Libre de Bruxelles, Campus Plaine, \\ Code Postal 231, B-1050 Brussels, Belgium \\ Received 14 February 2005; accepted 4 May 2005 \\ Available online 16 May 2005 \\ Communicated by A.R. Bishop
}

\begin{abstract}
A quantum master equation has been derived recently by Esposito and Gaspard [Phys. Rev. E 68 (2003) 066112] to study the dynamics of a quantum subsystem weakly interacting with another system which has a dense spectrum. This equation takes into account the fact that, because of energy conservation in the total system, the energy of the system with the dense spectrum is affected by the dynamics of the subsystem. We argue that such a situation can often be encountered in quantum nanosystems and we apply this equation to a nanosystem composed of a spin interacting with some remainder. By doing this, we demonstrate that quantum decoherence may already manifest itself in isolated nanosystems.
\end{abstract}

(C) 2005 Elsevier B.V. All rights reserved.

PACS: 05.30.-d; 03.65.Yz; 76.20.+q

Keywords: Quantum statistical mechanics; Decoherence; Relaxation; Thermalization; Master equation

A small quantum subsystem interacting with its environment undergoes irreversible processes such as relaxation and decoherence. Relaxation is the decay of the populations of the energy levels, while decoherence is the damping of the off-diagonal elements of the density matrix of the subsystem [2]. These decay processes are well known if the environment is an infinite thermal reservoir at a given temperature. Today, advances in nanoscience enable us to study isolated quantum systems of nanometric size containing a finite number of particles. In these nanosystems, a spin or a two-level subsystem may interact with the other degrees of freedom, which play the role of an environment and which we call the remainder of the system. In such isolated nanosystems, the total energy is conserved so that the dynamics takes place at fixed energy instead of fixed temperature.

\footnotetext{
* Corresponding author.

E-mail address: gaspard@ulb.ac.be (P. Gaspard).
} 


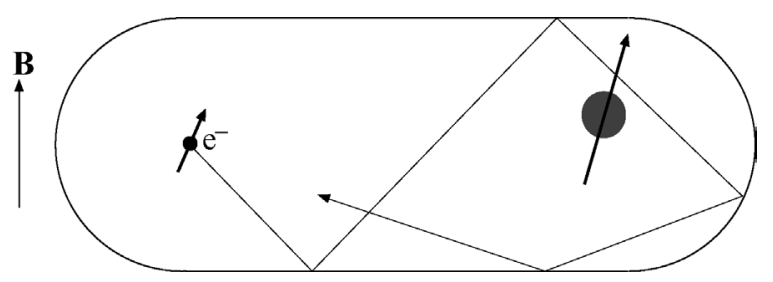

Fig. 1. Schematic representation of an electronic spin in a chaotic billiard quantum dot with a magnetic field perpendicular to the plane of the dot and interacting with a magnetic dipole-dipole potential due to a classical ferromagnetic impurity.

The aim of the present Letter is to show that the irreversible processes of relaxation and decoherence already emerge in such isolated nanosystems. An example is given by an electronic spin traveling in a semiconducting quantum dot with a chaotic billiard shape and submitted to a magnetic field. The quantum dot contains a localized ferromagnetic impurity with which a classical magnetic dipole is associated. The two spin levels of the traveling electron are split by the magnetic field and interact with the ferromagnetic impurity by the magnetic dipole-dipole potential. This system is schematically depicted in Fig. 1. Such devices are currently envisaged for understanding dissipation in quantum systems [3], as well as in the field of spintronics where spin relaxation and decoherence is ubiquitous [4]. Similar examples appear in the problem of decoherence of a qubit interacting with a couple of other qubits in devices for quantum information processing [5]. Related relaxation and decoherence processes are also observed in the dynamics of molecules or nanoclusters after their excitation by femtosecond laser pulses [6-8]. Such systems are described by the total Hamiltonian

$$
\hat{H}_{\mathrm{tot}}=\hat{H}_{\mathrm{s}}+\hat{H}_{\mathrm{r}}+\lambda \hat{S} \hat{R}
$$

where $\hat{H}_{\mathrm{S}}$ (respectively $\hat{H}_{\mathrm{r}}$ ) is the subsystem (respectively remainder) Hamiltonian and $\hat{S}$ (respectively $\hat{R}$ ) is the subsystem (respectively remainder) coupling operator.

We have derived in Ref. [1] a quantum master equation to describe the time evolution of the populations and coherences of a subsystem weakly interacting with the remainder of a total system where energy is conserved. This equation generalizes the weak-coupling master equations for the reduced density matrix of the subsystem (the socalled Redfield equations [9-12]). This generalization is performed by describing the time evolution as a function of the energy of the remainder. Our goal is to show that this master equation applies to quantum nanosystems and allows us to understand the decoherence processes which are internal to the nanosystem. For this purpose, we first summarize previous results.

The starting point of our description is the von Neumann equation ruling the time evolution of the density matrix of the total system. We suppose that the Hamiltonian $\hat{H}_{\mathrm{r}}$ of the remainder has a sufficiently dense spectrum that its level density is well represented by the smooth function $n(\epsilon)$. The central quantity of our theory is an operator acting on the subsystem state space and depending on the remainder energy $\epsilon$. It is defined as

$$
\hat{P}(\epsilon ; t) \equiv \operatorname{Tr}_{\mathrm{r}} \delta\left(\epsilon-\hat{H}_{\mathrm{r}}\right) \hat{\rho}(t),
$$

where $\hat{\rho}(t)$ is the density matrix of the total system. The reduced density matrix of the subsystem can be given in terms of this quantity as $\hat{\rho}_{\mathrm{S}}(t) \equiv \operatorname{Tr}_{\mathrm{r}} \hat{\rho}(t)=\int d \epsilon \hat{P}(\epsilon ; t)$. This shows that the quantity (2) distributes the populations and coherences of the subsystem over the energy $\epsilon$ of the remainder. The dynamics of the quantity $\hat{P}(\epsilon ; t)$ is ruled by an equation which is different from the usual perturbative master equation for the dynamics of the reduced density matrix $\hat{\rho}_{\mathrm{S}}(t)$ of the subsystem. For this reason, our equation keeps important information on the remainder time evolution. In order to get a closed evolution equation for the central quantity $\hat{P}(\epsilon ; t)$ of our theory, one needs to expands the complete von Neumann equation for the total system to second order in the coupling parameter $\lambda$ and use Eq. (2) [1,13]. One then gets the fundamental non-Markovian master equation of our theory 


$$
\begin{aligned}
\dot{\hat{P}}(\epsilon ; t)= & -i\left[\hat{H}_{\mathrm{s}}, \hat{P}(\epsilon ; t)\right] \\
& +\lambda^{2} \int_{0}^{t} d \tau \int d \omega\left\{-\tilde{\alpha}(\epsilon ;-\omega) \mathrm{e}^{i \omega \tau} \hat{S} \hat{S}(-\tau) \hat{P}(\epsilon ; t)-\tilde{\alpha}(\epsilon ;-\omega) \mathrm{e}^{-i \omega \tau} \hat{P}(\epsilon ; t) \hat{S}(-\tau) \hat{S}\right. \\
& \left.+\tilde{\alpha}(\epsilon-\omega ; \omega) \mathrm{e}^{i \omega \tau} \hat{S} \hat{P}(\epsilon-\omega ; t) \hat{S}(-\tau)+\tilde{\alpha}(\epsilon-\omega ; \omega) \mathrm{e}^{-i \omega \tau} \hat{S}(-\tau) \hat{P}(\epsilon-\omega ; t) \hat{S}\right\}
\end{aligned}
$$

The dynamics of $\hat{P}(\epsilon ; t)$ involves different values of the remainder energy, correlating the subsystem state with the remainder state. The coupling to the remainder is fully characterized by the Fourier transform of the microcanonical correlation function $\tilde{\alpha}(\epsilon ; \omega)=n(\epsilon+\omega) F(\epsilon, \epsilon+\omega)=\int_{-\infty}^{+\infty} \frac{d t}{2 \pi} \mathrm{e}^{i \omega t} \alpha(\epsilon ; t)$ defined as $\alpha(\epsilon ; t)=$ $\operatorname{Tr}_{\mathrm{r}} \hat{\rho}_{\mathrm{r}} \hat{R}(t) \hat{R}(0)=\int d \epsilon^{\prime} \mathrm{e}^{i\left(\epsilon-\epsilon^{\prime}\right) t} n\left(\epsilon^{\prime}\right) F\left(\epsilon, \epsilon^{\prime}\right)$, where $\hat{\rho}_{\mathrm{r}}=\delta\left(\epsilon-\hat{H}_{\mathrm{r}}\right) / n\left(\hat{H}_{\mathrm{r}}\right)$ and $F\left(\epsilon, \epsilon^{\prime}\right)=\overline{\left|\left\langle\epsilon|\hat{R}| \epsilon^{\prime}\right\rangle\right|^{2}}$. The overline denotes a smoothing over the dense spectrum of the remainder. We notice that the derivation of Eq. (3) requires to assume $\overline{\langle\epsilon|\hat{R}| \epsilon\rangle}=0$. Let us mention that $\pi \lambda^{2} \tilde{\alpha}(\epsilon ; \omega)$ is the Fermi golden rule transition probability rate of the remainder to jump from the remainder energy $\epsilon$ to the remainder energy $\epsilon+\omega$. By integrating Eq. (3) over the remainder energy, our equation reduces to the Redfield equation under the condition that the remainder correlation function $\tilde{\alpha}(\epsilon ; \omega)$ does not vary too much over energy scales of the order of the subsystem energies $\hbar \omega_{s, s^{\prime}}$ : $\tilde{\alpha}(\epsilon ; \omega) \simeq \tilde{\alpha}\left(\epsilon \pm \hbar \omega_{s, s^{\prime}} ; \omega\right)$. This condition is only satisfied when the remainder is large enough to be unaffected by the subsystem dynamics. In such cases the remainder becomes a heat bath. It is worth noting that this condition is similar to the requirement of equivalence between the microcanonical and the canonical ensembles [13].

We now consider a two-level system inside a nanosystem at fixed energy. We assume that the two-level system interacts in a non-diagonal way with the remainder of the nanosystem. We call this model the spin-remainder model. The total Hamiltonian is given by $\hat{H}_{\text {tot }}=\frac{\Delta}{2} \hat{\sigma}_{z}+\hat{H}_{\mathrm{r}}+\lambda \hat{\sigma}_{x} \hat{R}$. We remark that it has the same form as the famous spin-boson Hamiltonian [12]. The subsystem density matrix has the form

$$
\hat{\rho}_{\mathrm{S}}(t)=\int d \epsilon\left(\begin{array}{ll}
P_{++}(\epsilon ; t) & P_{+-}(\epsilon ; t) \\
P_{-+}(\epsilon ; t) & P_{--}(\epsilon ; t)
\end{array}\right) .
$$

Eq. (3) takes the simple form

$$
\begin{aligned}
\dot{P}_{ \pm \pm}(\epsilon ; t)= & -2 \lambda^{2} \int d \omega \tilde{\alpha}(\epsilon ;-\omega) P_{ \pm \pm}(\epsilon ; t) \frac{\sin (\omega \pm \Delta) t}{\omega \pm \Delta} \\
& +2 \lambda^{2} \int d \omega \tilde{\alpha}(\epsilon-\omega ; \omega) P_{\mp \mp}(\epsilon-\omega ; t) \frac{\sin (\omega \pm \Delta) t}{\omega \pm \Delta}
\end{aligned}
$$

for the distributions of the populations and

$$
\begin{aligned}
\dot{P}_{ \pm \mp}(\epsilon ; t)= & \mp i \Delta P_{ \pm \mp}(\epsilon ; t)-\lambda^{2} \int d \omega \tilde{\alpha}(\epsilon ;-\omega) P_{ \pm \mp}(\epsilon ; t) \int_{0}^{t} d \tau\left[\mathrm{e}^{i(\omega \pm \Delta) \tau}+\mathrm{e}^{-i(\omega \mp \Delta) \tau}\right] \\
& +\lambda^{2} \int d \omega \tilde{\alpha}(\epsilon-\omega ; \omega) P_{\mp \pm}(\epsilon-\omega ; t) \int_{0}^{t} d \tau\left[\mathrm{e}^{i(\omega \mp \Delta) \tau}+\mathrm{e}^{-i(\omega \pm \Delta) \tau}\right]
\end{aligned}
$$

for the distributions of the coherences. These equations are non-Markovian because of the time integral in the right-hand side. We notice that the non-Markovian Eqs. (5) and (6) couple together the distributions $\hat{P}(\epsilon ; t)$ at different remainder energies. This means that the distributions of populations or coherences, if initially localized at a given remainder energy, can spread over the remainder energy during the time evolution. For time scales longer than the remainder correlation time (typically the time over which $\alpha(\epsilon ; t)$ goes to zero), we can use the Markovian approximation, that consists in putting the upper bound of the time integral to infinity. The Markovian population 
dynamics is given by

$$
\dot{P}_{ \pm \pm}(\epsilon ; t)=-2 \pi \lambda^{2} \tilde{\alpha}(\epsilon ; \pm \Delta) P_{ \pm \pm}(\epsilon ; t)+2 \pi \lambda^{2} \tilde{\alpha}(\epsilon \pm \Delta ; \mp \Delta) P_{\mp \mp}(\epsilon \pm \Delta ; t)
$$

and the Markovian coherence dynamics by

$$
\dot{P}_{ \pm \mp}(\epsilon ; t) \simeq\left[\mp i(\Delta-\Gamma)-\gamma_{\mathrm{coh}}\right] P_{ \pm \mp}(\epsilon ; t),
$$

where $\gamma_{\mathrm{coh}}=\pi \lambda^{2}[\tilde{\alpha}(\epsilon ; \Delta)+\tilde{\alpha}(\epsilon ;-\Delta)]$ and $\Gamma=2 \Delta \lambda^{2} \int d \omega \mathcal{P} \frac{\tilde{\alpha}(\epsilon ; \omega)}{\omega^{2}-\Delta^{2}}$. The Markovian dynamics can be well described in terms of the quantities

$$
\begin{aligned}
& C(\epsilon ; t) \equiv P_{++}(\epsilon ; t)+P_{--}(\epsilon+\Delta ; t), \\
& Z(\epsilon ; t) \equiv P_{++}(\epsilon ; t)-P_{--}(\epsilon+\Delta ; t), \\
& X(\epsilon ; t) \equiv P_{+-}(\epsilon ; t)+P_{-+}(\epsilon ; t), \\
& Y(\epsilon ; t) \equiv i P_{+-}(\epsilon ; t)-i P_{-+}(\epsilon ; t),
\end{aligned}
$$

where $C(\epsilon ; t)$ and $Z(\epsilon ; t)$ characterize the subsystem populations and $X(\epsilon ; t)$ and $Y(\epsilon ; t)$ the subsystem coherences. Using Eqs. (7) and (8), one gets

$$
\begin{aligned}
& C(\epsilon ; t)=C(\epsilon ; 0), \\
& Z(\epsilon ; t)=Z(\epsilon ; \infty)+[Z(\epsilon ; 0)-Z(\epsilon ; \infty)] \mathrm{e}^{-\gamma_{\mathrm{pop}} t}, \\
& X(\epsilon ; t)=[X(\epsilon ; 0) \cos (\Delta-\Gamma) t-Y(\epsilon ; 0) \sin (\Delta-\Gamma) t] \mathrm{e}^{-\gamma_{\mathrm{coh}} t}, \\
& Y(\epsilon ; t)=[X(\epsilon ; 0) \sin (\Delta-\Gamma) t+Y(\epsilon ; 0) \cos (\Delta-\Gamma) t] \mathrm{e}^{-\gamma_{\mathrm{coh}} t},
\end{aligned}
$$

where $\gamma_{\text {pop }}=2 \pi \lambda^{2}[\tilde{\alpha}(\epsilon ; \Delta)+\tilde{\alpha}(\epsilon+\Delta ;-\Delta)]$ and $Z(\epsilon ; \infty)=\frac{n(\epsilon)-n(\epsilon+\Delta)}{n(\epsilon)+n(\epsilon+\Delta)} C(\epsilon ; 0)$. The two-level subsystem dynamics is fully described in terms of these quantities because $w(t)=\operatorname{Tr} \hat{\rho}(t) \hat{\sigma}_{w}=\int d \epsilon^{\prime} W\left(\epsilon^{\prime} ; t\right)$, where $w=x, y, z$ and $W=X, Y, Z$. If we choose the initial condition as the product of a general subsystem density matrix with a microcanonical distribution at energy $\epsilon$ for the remainder, $\hat{P}\left(\epsilon^{\prime} ; 0\right)=\hat{\rho}_{\mathrm{s}}(0) \delta\left(\epsilon^{\prime}-\epsilon\right)$, the two-level subsystem has a biexponential relaxation for the populations and an oscillatory exponential relaxation for the coherences:

$$
z(t)=Z(\epsilon ; t)+Z(\epsilon-\Delta ; t), \quad x(t)=X(\epsilon ; t), \quad y(t)=Y(\epsilon ; t) .
$$

Indeed, the populations decay at the rates $\gamma_{\text {pop }}(\epsilon)$ and $\gamma_{\text {pop }}(\epsilon-\Delta)$ and the coherences at the rate $\gamma_{\text {coh }}(\epsilon)$. In contrast to the usual approach where the remainder is a heat bath, we here have, in isolated nanosystems, two relaxation rates for the populations instead of one. Each of these rates corresponds to a pair of remainder energies, shifted one with respect to the other by an amount $\Delta$, and between which the system undergoes transitions.

In order to illustrate the time evolution of the distribution functions $\hat{P}(\epsilon ; t)$ of the subsystem, we consider a spinremainder model in which the remainder operators are taken as Gaussian orthogonal random matrices (GORM). The motivation for such an assumption is that in some nanosystems, the degrees of freedom playing the role of the remainder may be classically chaotic and form a complex quantum system. In such systems, some statistical properties can be modeled by random matrices according to the Bohigas-Giannoni-Schmit conjecture [14]. Such assumptions are considered for nanosystems [15]. In the example of Fig. 1, the spatial motion of the electron is classically chaotic and its Hamiltonian operator as well as the other operators of the coupling with the magnetic field can therefore be modeled by random matrices. For this so-called spin-GORM model, $\hat{H}_{\mathrm{r}}=\frac{1}{\sqrt{8 N}} \hat{X}$ and $\hat{R}=$ $\frac{1}{\sqrt{8 N}} \hat{X}^{\prime}$, where $\hat{X}$ and $\hat{X}^{\prime}$ are two statistically independent $\frac{N}{2} \times \frac{N}{2}$ random matrices. The elements of $\hat{X}$ and $\hat{X}^{\prime}$ are independent Gaussian random real numbers with mean zero and standard deviations 1 for the off-diagonal elements, and $\sqrt{2}$ for the diagonal elements. $N / 2$ is the number of states of the remainder and is here assumed large. Using the convention $\sqrt{x} \equiv 0$ if $x \leqslant 0$, we find for the spin-GORM model that $n(\epsilon)=\frac{4 N}{\pi} \sqrt{1 / 4-\epsilon^{2}}$ and $F\left(\epsilon, \epsilon^{\prime}\right)=\frac{1}{8 N}$, and therefore that $\tilde{\alpha}(\epsilon ; \omega)=\frac{1}{2 \pi} \sqrt{1 / 4-(\epsilon+\omega)^{2}}$. Notice that the width of the density of states of 

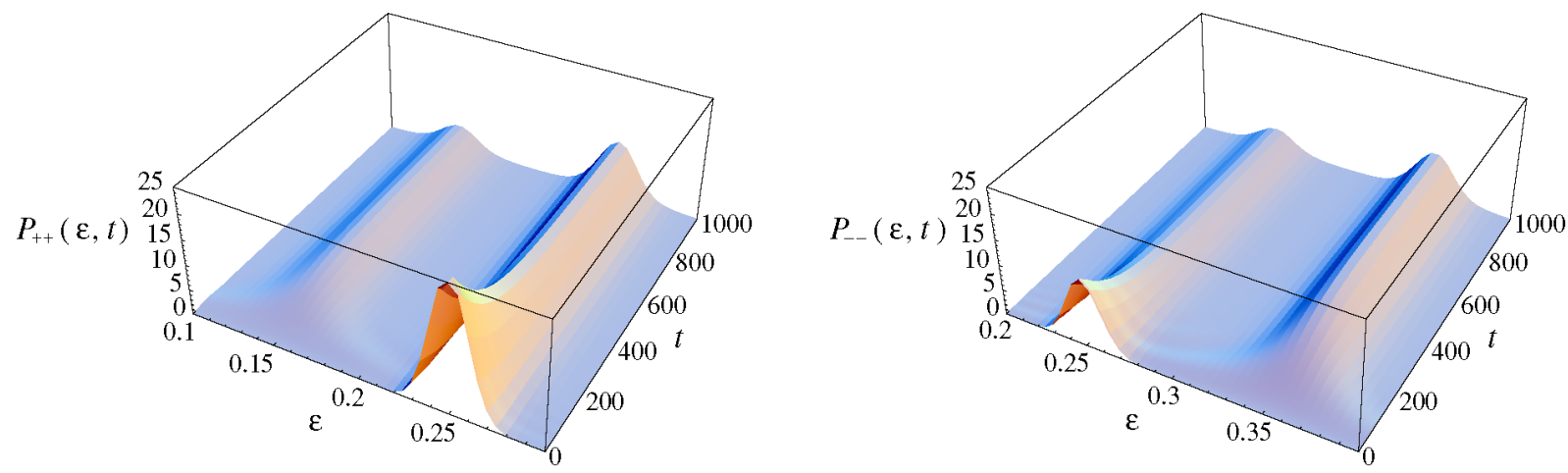

Fig. 2. Time evolution of the populations $P_{++}(\epsilon ; t)$ and $P_{--}(\epsilon ; t)$ for $\Delta=0.1025, \epsilon=0.25, \lambda=0.1$.
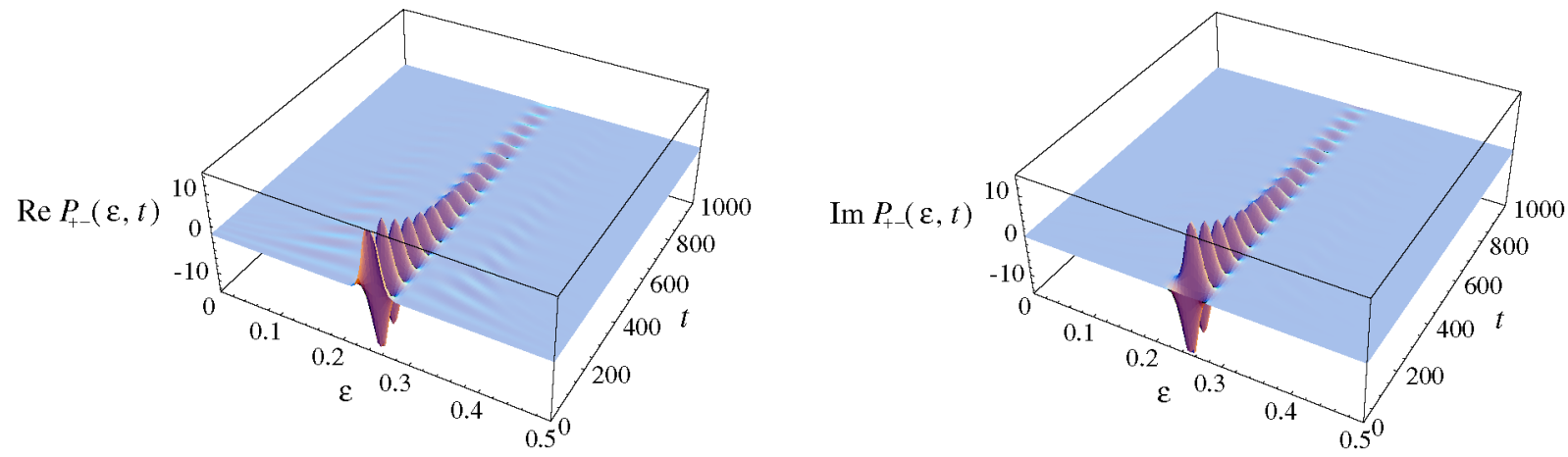

Fig. 3. Time evolution of the real and imaginary parts of $P_{+-}(\epsilon ; t)$ for $\Delta=0.1025, \epsilon=0.25, \lambda=0.1$. The small features in the wings of the real part are due to non-Markovian effects.

the remainder is not affected by the limit of large $N$, so that the limit of a dense spectrum does not correspond to the limit of a remainder with an infinite number of degrees of freedom and is thus compatible with the fact that the total system is a nanosystem. Similar models have been studied in $[3,6,13,16-20]$.

In the spin-GORM model, the non-Markovian dynamics of $\hat{P}(\epsilon ; t)$ can be studied by numerical integration of Eqs. (5) and (6). The numerical results are depicted in Figs. 2 and 3, where the initial condition of the system is the pure state $\hat{\rho}_{\mathrm{S}}(0)=\left(\begin{array}{cc}2 / 3 & \sqrt{2} / 3 \\ \sqrt{2} / 3 & 1 / 3\end{array}\right)$. The initial distribution of the remainder energy is taken as a Gaussian centered at $\epsilon=0.25$ and with standard deviation 0.01 . We observe in Fig. 2, that the probability disappearing in $P_{++}(\epsilon ; t)$ reappears in $P_{--}(\epsilon+\Delta ; t)$ due to the closed dynamics of $Z(\epsilon ; t)$ on the total energy shell $\frac{\Delta}{2}+\epsilon$ and, similarly, for the probability disappearing in $P_{--}(\epsilon+\Delta ; t)$ and reappearing in $P_{++}(\epsilon ; t)$ on the total energy shell $-\frac{\Delta}{2}+\epsilon$. In Fig. 3, we see that the distribution of the coherences has an oscillating decay essentially on the initial remainder energy shell with very small non-Markovian effects at other energies. The decays of Figs. 2 and 3 predicted by our quantum master equation (3) are in excellent agreement with the decays calculated with the exact von Neumann equation [13].

In conclusion, we have shown that relaxation and decoherence can already emerge in finite quantum systems such as a nanosystem made of a subsystem interacting with the remainder of the nanosystem. In such systems energy is conserved and dissipation only occurs between the subsystem and the other degrees of freedom constituting the remainder. The description of such irreversible processes can be carried out thanks to a special quantum master equation which rules the distributions of the populations and coherences of the subsystem over the energy of the remainder. This master equation describes the exchange of energy between the subsystem and the remainder in 
agreement with the exact dynamics and generalizes the approaches where the remainder is considered as a heat bath. We showed that the subsystem populations relax according to a biexponential as a consequence of the total energy conservation (although the heat bath approach predicts a single exponential decay). Moreover, the quantum coherences can also be distributed on the energy of the remainder and their time evolution presents an oscillatory damping characteristic of decoherence with small additional features due to non-Markovian effects. We believe that this new way of describing the time evolution of a quantum subsystem inside a nanosystem can be of interest for understanding recent experiments in nanotechnology.

\section{Acknowledgements}

The authors thank Professor G. Nicolis for support and encouragement in this research. M.E. is supported by the "Ministère de la Culture, de l'Enseignement Supérieur et de la Recherche du Grand-Duché de Luxembourg". This research is financially supported by the "Communauté française de Belgique" ("Actions de Recherche Concertées", contract No. 04/09-312), the National Fund for Scientific Research (F.N.R.S. Belgium), the F.R.F.C. (contracts Nos. 2.4542.02 and 2.4577.04), and the U.L.B.

\section{References}

[1] M. Esposito, P. Gaspard, Phys. Rev. E 68 (2003) 066112.

[2] W.H. Zurek, Rev. Mod. Phys. 75 (2003) 715.

[3] D. Cohen, T. Kottos, Phys. Rev. E 69 (2004) 055201(R).

[4] I. Zutic, J. Fabian, S. Das Sarma, Rev. Mod. Phys. 76 (2004) 323.

[5] G. Benenti, G. Casati, D. Shepelyansky, Eur. Phys. J. D 17 (2001) 265.

[6] W.M. Gelbart, S.A. Rice, K.F. Freed, J. Chem. Phys. 57 (1972) 4699.

[7] R.A. Marcus, Adv. Chem. Phys. 101 (1997) 391.

[8] Y. Jung, E. Barkai, R.J. Silbey, Adv. Chem. Phys. 123 (2002) 199.

[9] A.G. Redfield, IBM J. Res. Dev. 1 (1957) 19.

[10] C.W. Gardiner, P. Zoller, Quantum Noise, second ed., Springer-Verlag, Berlin, 2000.

[11] R. Kubo, M. Toda, N. Hashitsume, Statistical Physics II: Nonequilibrium Statistical Mechanics, second ed., Springer-Verlag, Berlin, 1998.

[12] P. Gaspard, M. Nagaoka, J. Chem. Phys. 111 (1999) 5668.

[13] M. Esposito, PhD Thesis, cond-mat/0412495.

[14] O. Bohigas, M.J. Giannoni, C. Schmit, Phys. Rev. Lett. 52 (1984) 1.

[15] W.P. Halperin, Rev. Mod. Phys. 58 (1986) 533.

[16] M. Esposito, P. Gaspard, Phys. Rev. E 68 (2003) 066113.

[17] P.A. Mello, P. Pereyra, N. Kumar, J. Stat. Phys. 51 (1988) 77.

[18] P. Pereyra, J. Stat. Phys. 65 (1991) 773.

[19] E. Lutz, H.A. Weidenmüller, Physica A 267 (1998) 354.

[20] J.L. Lebowitz, L. Pastur, J. Phys. A 37 (2004) 1517. 